\title{
Autonomia e integração dos usuários transexuais em estratégia de saúde da família
}

\section{Autonomy and integration of transexual users in family health strategy}

\author{
Denildo de Freitas Gomes' $\bullet$ Enéas Rangel Teixeira ${ }^{2} \bullet$ Marta Sauthier $^{3} \bullet$ Donizete Vago Daher ${ }^{4}$ \\ Rafael Gravina Fortini ${ }^{5}$ Jaqueline da Silva Soares Souto
}

\begin{abstract}
RESUMO
Objetivos: identificar as publicações indexadas em banco de dados; caracterizar essas produções e analisar os resultados com apoio na teoria do reconhecimento honnetiana, oferecendo uma síntese dessas publicações. Metodologia: Revisão integrativa da literatura sistematizada, qualitativa.A questão constituiu-se em quais são as evidências sobre vulnerabilidades e integração dos usuários transexuais para seu reconhecimento nos cuidados em saúde? Recorte de 2012 a 2018 nos bancos de dados da BVS e PubMed com DECs e Mesh, respectivamente. A consulta fora realizada em 10 de agosto de 2017. Resultados: Foram encontrados 55 artigos e após utilização dos filtros, com critérios de inclusão e exclusão, restaram 25 artigos, emergindo três categorias, a saber, paradigma biomédico; estigma, invisibilidade e heteronomia; processo de trabalho.As discussões versaram sobre modelo biomédico e invisibilidade desses usuários estigmatizados, perpassando pelo processo de trabalho deficitário, por falta de conhecimentos sobre questões socioculturais de gênero e técnicas. Conclusão:A realidade descrita é inversa à integralidade da assistência que se deseja, distante da equidade pretendida, sensíveis à vulnerabilidade da população em estudo. Esses usuários, concebidos com rótulos pré-determinados, são sujeitos integrantes da comunidade em que a aceitação e convívio dependem de mudança de paradigma, em que a assistência/cuidado recebido contribua para o reconhecimento desses usuários.
\end{abstract}

Descritores: Atenção primária à saúde;Transexualismo; Pessoas transgêneros.

\begin{abstract}
Objectives: to identify publications indexed in database; characterize these productions and analyze the results with support in the theory of Honnetian recognition, offering a synthesis of these publications. Methodology: Integrative review of systematized, qualitative literature. The question consisted in what are the evidences about vulnerabilities and integration of transsexual users for their recognition in health care? Cut from 2012 to 2018 in the VHL and PubMed databases with DECs and Mesh, respectively. The study was carried out on August 10, 2017. Results: 55 articles were found and after the use of the filters, with inclusion and exclusion criteria, there were 25 articles, three categories emerged, namely biomedical paradigm; stigma, invisibility and heteronomy; work process. The discussions focused on the biomedical model and invisibility of these stigmatized users, passing through the process of deficit work, lack of knowledge about gender and socio-cultural issues and techniques. Conclusion:The reality described is inverse to the integrality of the assistance that is desired, far from the desired equity, sensitive to the vulnerability of the study population. These users, designed with pre-determined labels, are subjects of the community in which the acceptance and conviviality depend on paradigm change, in which the care / care received contributes to the recognition of these users.
\end{abstract}

Keywords: Primary health care;Transsexualism; Transgender people.

'Mestre em Ciências do Cuidado da Saúde no Contexto Sociocultural pela Escola de Enfermagem Aurora de Afonso Costa -Universidade Federal Fluminense (EEAC-UFF); Professor Substituto da Escola de Enfermagem Anna Nery (EEAN)- Universidade Federal do Rio de Janeiro(UFRJ).

${ }^{2}$ Doutor em enfermagem pela Universidade Federal do Rio de Janeiro (UFRJ); Pós-doutorado em Psicologia Clínica pela Pontifícia Universidade Católica (PUC) - São Paulo; Professor titular da Universidade Federal Fluminense (UFF).

${ }^{3}$ Doutora em Enfermagem pela Universidade Federal do Rio de Janeiro (UFRJ); Docente Associada I da Escola de Enfermagem Anna Nery (EEAN)

${ }^{4}$ Doutora em Saúde Coletiva pela Universidade Estadual de Campinas (FCM-UNICAMP); Pós-doutorado com dupla titulação: FE/UERJ e ESEnFC/Coimbra/Pt; Docente Titular da Universidade Federal Fluminense.

${ }^{5}$ Mestre em Ciências do Cuidado da Saúde no Contexto Sociocultural pela Universidade Federal Fluminense (UFF); Doutorando em Ciências do Cuidado em saúde pela Escola de Enfermagem Aurora de Afonso Costa - Universidade Federal Fluminense (EEAAC - UFF).

${ }^{6}$ Mestranda do Programa de Pós-graduação em enfermagem da Universidade Federal do Rio de Janeiro (UFRJ); Professora Substituta da Escola de Enfermagem Anna Nery (EEAN)- Universidade Federal do Rio de Janeiro (UFRJ). 


\section{INTRODUÇÃO}

Dentro da saúde coletiva, diferente da saúde pública, as mudanças em favor da comunidade conforme suas necessidades vão além de diagnósticos ou da visão biomédica, positivista ou cartesiana, exigindo dos profissionais uma visão mais abrangente. Com essa perspectiva, as subjetividades e questões sociais e políticas interferem nas respostas humanas e acessos à saúde, bem como, na integração dos usuários na rede de saúde, convergentes com as diretrizes do Sistema Único de Saúde (SUS). Nesse pensamento, em consonância com a equidade e integralidade preconizada pelo SUS, criou-se o Programa de Saúde da Família (PSF). Esse programa, por ser realizado em tempo determinado e por sua ideologia, evoluiu para a Estratégia de Saúde da Família (ESF), criada em 2006, ampliando essa concepção por ser permanente e contínua ${ }^{(1)}$.

Nesse contexto contemporâneo da estratégia de saúde da família, tornam-se desafiadoras relações de atendimento aos usuários transexuais, definidos de forma contrária à concepção anterior de patogenicidade. Observam-se limitações em serviços individualizados e subjetivados para a demanda das especificidades biopsicossociais, sendo importante reconhecer que, dentro do processo social saúde-doença, o estigma e o preconceito acabam por excluir determinados grupos, estando entre eles os transgêneros e, dentro desse grupo, os transexuais.

No Brasil, não há um consenso sobre o termo transgêneros. Esses são caracterizados pelas diversas formas de viver o gênero a que se identificam. Como definição, a mulher transexual é toda aquela pessoa que reivindica o reconhecimento como mulher, assim como o homem transexual reivindica o reconhecimento como homem. Esse processo pode ou não passar pela transgenitalização, que é a reconstrução cirúrgica dos genitais a que o indivíduo se identifica e pela hormonização. Em sentido lato, transexual é o indivíduo que possui uma identidade de gênero oposta ao sexo designado (normalmente no nascimento). $O$ termo transgênero tem sido definido como ruptura entre as determinações sociais e culturais relacionadas ao sexo e entre o desejo ou subjetividade dos sujeitos, favorecendo as diferentes expressões de identidade de gênero. Quanto ao termo transexual, a definição inclui a efetiva cirurgia de transgenitalização ${ }^{(2)}$.

Homens e mulheres transexuais fazem ou pretendem fazer uma transição de seu sexo de nascimento para o sexo oposto (sexo-alvo) com alguma ajuda médica (terapia de redesignação de sexo) para seu corpo. Especificamente, transgenitalização consiste nos procedimentos cirúrgicos denominados neocolpovulvoplastia e neofaloplastia. A transgenitalização permite a mudança do aparelho sexual, importando apenas alterações estéticas e não genéticas ${ }^{(3)}$.

A exclusão das pessoas transgêneros e transexuais abrange não só o acesso à saúde, mas também o direito a emprego, moradia e educação. Considerando vulnerabilidade diretamente relacionada à diminuição da autonomia, conforme a perspectiva honnethiana, pode-se definir vulneráveis como indivíduos que sofrem danos à sua autonomia por relações sociais desiguais. Na perspectiva Honnethiana, o ser livre para tomar decisões é um ser autônomo. Entretanto essa liberdade é oriunda de responsabilização do indivíduo por suas decisões e ações, sendo esse ser livre de coerções. Ainda nessa perspectiva, a heteronomia pode ocorrer, por exemplo, a partir do momento em que as decisões dos indivíduos encontramse obliteradas por falta de orientação de profissionais para tomada de decisão. ${ }^{(4)}$

Nesse sentido, todos, em algum momento, nos tornamos vulneráveis, entretanto os transexuais encontramse em situação de maior vulnerabilidade: "para que seja produzido o sujeito vulnerável, é necessário que esta série de discursos e dispositivos de poderes e preocupações do governo seja acionada, tornando indivíduos legítimos às políticas públicas e dignos de seus direitos humanos"(5).

A percepção dos profissionais sobre a identificação de gênero repercute na assistência prestada, que, por vezes, tratada com distanciamento dos direitos legítimos desses usuários, tais como, ser identificado pelo nome social, tratar as especificidades clínicas, especialmente tratamentos hormonais e acompanhamento de saúde mental, entre outros, exigem profissionais atentos e capacitados para o atendimento a esse grupo.

Nossa observação na prática em ESF aponta para uma diminuição na procura de atendimento por parte dos usuários transexuais, confirmadas pela afirmação de alguns deles, referindo não se sentirem integrados ao serviço oferecido pela Estratégia de Saúde da Família. Com isso, o problema do estudo são os déficits no processo de trabalho multidisciplinar em saúde, preconceitos ou invisibilidade/exclusão sobre usuários transexual em ESF.A questão construída constitui-se em quais são as evidências sobre vulnerabilidades e integração dos usuários transexuais para seu reconhecimento nos cuidados em saúde? O objeto do estudo foi o reconhecimento dos usuários transexuais na assistência em saúde. Os objetivos construídos foram: identificar as publicações indexadas em banco de dados; caracterizar essas produções e analisar os resultados apoiados na teoria do reconhecimento honnethiana.

Tal revisão justifica-se pela necessidade de elucidar subjetividades, relacionadas a discussões de gênero e apontar caminhos para a visibilidade e o reconhecimento dos usuários transexuais, como cidadãos de direitos, a serem considerados nas suas especificidades na assistência em saúde. Esse estudo será capaz de trazer à tona fundamentos e conceitos significativos para a atualização 
sobre o reconhecimento das pessoas transexuais assistido na Estratégia de Saúde Família ou em qualquer esfera de atendimento do sistema de saúde. Além disso, contribuir com os usuários na disseminação em nível nacional e internacional, esclarecimentos de conceitos e estratégias de atendimento singularizado e especializado, em que se vislumbre principalmente o estabelecimento de uma relação empática e transpessoal entre profissional de saúde-pessoa transexual.

Os profissionais que acessarem esta publicação e as próprias instituições de saúde poderão sensibilizar-se quanto à necessidade de (re)avaliar ou considerar os cuidados com os usuários em tela. Principalmente, após a proposta HumanizaSUS, bem como da Política de Assistência Integral à saúde LGBT, ou políticas estabelecidas em outros países, tornando essa proposta uma meta para instituições e profissionais.

Propositalmente, a Teoria do Reconhecimento honnetiana aparece como 'pano de fundo', trazendo sustentação teórica aos resultados encontrados na síntese dos artigos acessados. Para tanto, explicitar-se-á, a seguir, conceitos e a própria teoria para a leitura das produções e o pensamento acerca dos direitos dos cidadãos, em que pese o reconhecimento e efetividade do exercício da autonomia. $\mathrm{O}$ autor, pertencente à escola de Frankfourt, mais especificamente, à Escola da Teoria Crítica, explicita que na base da interação está o conflito social, construindo sua teoria, em que a gramática é a própria luta por reconhecimento. Assim, configura o conflito como agente fundamental para a socialização e emancipação humana, não considerando luta social como luta pelo poder ou autoconservação, mas sim, como forma de reconhecimento por identidades individuais e coletivas, frente às ações de desrespeito social. $O$ desrespeito interfere não somente na imobilidade ou paralisia de um indivíduo, mas também na estagnação de um grupo social, tornando-o invisível ou excluído e "dependente do reconhecimento social". Nesse sentido, a luta por reconhecimento contribui como "modelo de interpretação de lutas sociais e processo de desenvolvimento moral" (6).

A teoria em tela apresenta $\circ$ reconhecimento nas esferas do amor, da solidariedade e do direito. Especialmente, apresenta o reconhecimento em três dimensões, a saber, a autoconfiança, conquistada ainda quando criança, junto à mãe; o autorrespeito, que pode ser formada por escolha entre normas e, por último, a valoração social, que se refere às qualidades individuais ou diferenciais. Considerando as esferas do amor, do respeito, estima e sua aplicação nas relações sociais, percebe-se o desenvolvimento de uma atitude positiva do sujeito, garantindo-lhe um sentimento de autorrespeito e autoestima podendo, assim, identificar e concretizar seus objetivos e desejos.
Nesse pensamento, o usuário transexual, assim como os demais cidadãos necessitam de respeito, contribuição no exercício da autonomia, alteridade e estima/solidariedade, o que se contrapõe, em uma concepção dialética, ao desrespeito, heteronomia e desconsideração/invisibilidade. Na perspectiva Honnethiana, o ser livre para tomar decisões é um ser autônomo. Entretanto essa liberdade é oriunda de responsabilização do indivíduo por suas decisões e ações, sendo esse ser livre de coerções. Ainda nessa perspectiva, a heteronomia pode ocorrer, por exemplo, a partir do momento em que as decisões dos indivíduos encontram-se obliteradas por falta de orientação de profissionais para tomada de decisão.

\section{MÉTODO}

Estudo de revisão integrativa sistematizada da literatura de natureza qualitativa, do tipo descritivo, conforme Minayo $^{(7)}$, na pesquisa descritiva, o estudo do tema, assim como a análise, registro e interpretação dos fatos ocorrem sem a inferência do autor, cabendo-lhe apenas descrever fatos, neste caso, já indexados por outros autores. Ou seja, convergente com a revisão integrativa, proporcionando a síntese de conhecimento e a incorporação de resultados de estudos publicados, significativos na prática, a serem considerados para a utilização desse recurso metodológico. A natureza qualitativa desse artigo se dá pela importância em dar foco aos fatos, causas ou fenômenos socioculturais ${ }^{(8-7)}$.

A revisão integrativa sistematizada desenvolveu-se ao longo das seis etapas que a constituiu. Na primeira etapa, identificou-se o tema e seleção da questão fundamental para a elaboração desta pesquisa, com uso da estratégia PIO, que se segue: Quais são as evidências sobre vulnerabilidades, tais como abjeção do corpo e integração dos usuários transexuais para seu reconhecimento nos cuidados em saúde? O corpo abjeto é o corpo rechaçado, descrito, mas não percebido no cotidiano e, consequentemente, não priorizado ou considerado no cuidado em saúde, tema que remete à questão referida ${ }^{(9)}$.

$\mathrm{Na}$ segunda etapa, estabeleceram-se critérios para inclusão, tais como, artigos científicos publicados nos últimos seis anos (a partir de agosto de 2012 até agosto de 2018), em língua inglesa, espanhola e portuguesa, artigos completos e em revistas indexadas em banco de dados da área da saúde, especialmente em enfermagem, que abordassem o tema transexualidade em humanos, constantes na Biblioteca Virtual de Saúde (BVS) e Centro Nacional para a Informação Biotecnologia (PUBMED), tais como índice da Literatura Latino-Americana e do Caribe em Ciências da Saúde (LILACS), Base de dados de enfermagem (BDENF) e (Medical Literature Analysis and Retrieval System Online) que é a base de dados bibliográficos da Biblioteca Nacional de Medicina dos Estados 
Unidos da América (MEDLINE), respectivamente, que tratassem sobre o tema de pesquisa. Os descritores em Ciências da Saúde (DECs) foram transexualismo, pessoas transgênero e atenção primária à saúde. $O$ Medical Subject Headings ou sistema de metadados médicos (MESH) foram transsexualism, transgender persons e primary helth care. Os critérios de exclusão foram artigos repetidos, Anais de eventos ou resumos simplificados e expandidos, teses, dissertações e artigos ou textos de blogs e sites de saúde.

Em sequência, iniciou-se a busca na BVS e PUBMED, as publicações indexadas sofreram aplicação dos filtros e refinamento com boleano and. Após a pré-seleção, que reuniu 109 publicações, realizou-se a identificação dos artigos repetidos, que foram I8, restando 9/ artigos para leitura. Após leitura atentiva e pormenorizada dos artigos na íntegra, obteve-se 25 artigos com enfoque no tema dessa pesquisa, a saber, aspectos que interferem nos cuidados em saúde aos usuários transexuais, que foram selecionados e organizados na Tabela I, intitulada: Distribuição dos artigos por ano de publicação, revista de publicação, profissionais autores, tipo de pesquisa, tipo de estudo, instituição e estado, de 2012 a 2017 , nacional e internacional.

A terceira etapa constituiu-se da definição de informações a serem extraídas dos artigos selecionados, seguida pela quarta etapa, cuja avaliação dos artigos, incluídos na busca, envolveu uma leitura prévia, sendo integralmente organizados na tabela I dos resultados dos artigos acessados. Após a síntese dos resultados dos artigos acessados e sua interpretação( ${ }^{(10)}$, reunindo esses resultados em núcleos de conteúdo, seguindo a técnica de análise de conteúdo de Bardin, finalizou-se esta quinta etapa, partindo-se para a sexta e última etapa desta pesquisa, a saber, apresentação dessa metassíntese. No método de revisão integrativa, não basta mostrar a síntese das produções, sendo necessário realizar análise crítica desses resultados.

\section{RESULTADOS}

As publicações, em 2012, ainda estavam tímidas, obtendo-se apenas $4 \%$ das produções. Apenas em 2013 houve um aumento, com $16 \%$, havendo uma nova redução nas publicações de $08 \%$ em 20I4. Em 2015 houve novo aumento de publicações, com I $2 \%$, tendo, em 2016 , alcançado o maior índice, com $48 \%$, caindo para $12 \%$ em 2017 até a data da última busca, mostrando que houve menor interesse em publicações sobre transgênero/ transexuais, ou por parte das revistas, ou por parte dos autores naquele período. $O$ que nos leva a ratificar a necessidade de se ampliar as divulgações sobre as pesquisas acerca do tema. Quanto mais pesquisas e divulgações forem realizadas, mais profundo será o conhecimento e maiores serão aceitação e atenção em saúde aos usuários transgênero, repercutindo em possível diminuição do estigma e provável melhora da capacitação dos profissionais de saúde na atenção básica. Vejamos a tabela seguinte, oriunda desta revisão integrativa da literatura sistematizada.

Quanto às revistas em que foram publicados os artigos, de todas as referidas, a revista que mais publicou sobre o tema foi a AMA Journal of Ethics e Curr Opin Endocrinol Diabetes Obes. com $32 \%$ das publicações, seguidas pelo Journal PLos One com 08\%, BMC Public Health com $08 \%$ e Arch Sex Behav com 08\% , tendo os outros periódicos somado $44 \%$ das publicações. A publicação maior em revistas com paradigma biomédico nos remete ao foco da atenção ao modelo referido, com temas focados principalmente em estudos sobre a administração de hormônios e seus efeitos adversos, indo até as questões éticas de consentimento informado como garantias legais que os respaldem em relação aos procedimentos realizados, sendo as abordagens de questões subjetivas periféricas, pouco desenvolvidas ou inexistentes.

Os profissionais que se dedicaram às publicações acessadas foram Médicos em $64 \%$ das ocorrências e sociólogos e psicólogos, com respectivamente, $8 \%$ cada. Dessas publicações, os demais autores foram advogados, Filósofo, Antropólogo, Terapeutas Ocupacionais e enfermeiros, com $4 \%$ das publicações cada um, totalizando $100 \%$ das publicações.

A abordagem qualitativa foi o tipo de pesquisa mais utilizado com $44 \%$ em relação à quantitativa, que totalizou $36 \%$, trazendo à tona algumas tendências nas áreas que mais publicaram, quais sejam, sobre compreensão do modo de vida, qualidade da assistência e aspectos sociais e subjetividades humanas, a saber, sociólogos e psicólogos. A participação de médicos emergiu com maior número de publicações, porém mais direcionadas às questões cirúrgicas e hormonais, ainda que já desvelem interesse em questões mais subjetivas em alguns artigos. A abordagem quantitativa foi composta por autores das áreas de medicina em sua grande maioria, $08 \%$ das produções eram de metodologia mista e outros $12 \%$.

Quanto ao tipo de pesquisa, $16 \%$ eram revisões integrativas sobre o tema e $32 \%$ eram revisões sistemáticas, $12 \%$ eram estudos do tipo revisão bibliográfica e estudo clínico, que se constituiu em $12 \%$, estudo etnográfico, em $08 \%$, artigos reflexivos, em $4 \%$, baseado em evidências, $8 \%$ e relato de experiência, em $8 \%$.

Com relação aos locais que mais publicaram sobre o tema transexualidade, obteve-se no Canadá e nos EUA, $24 \%$ das publicações. $O$ total de artigos acessados nos EUA foi de $68 \%$, no Brasil e Reino Unido o número de publicações foram 08\%, seguido do Novo México, Chile, Porto Rico e Japão, com 04\% de publicações respectiva- 
TABELA 1 - Distribuição dos artigos por ano de publicação, revista de publicação, profissionais, autores, tipo de pesquisa, tipo de estudo, instituição e estado, de 2012 a 2017, nacional e internacional - Niterói, RJ, Brasil, 2017.

\begin{tabular}{|c|c|c|}
\hline \multirow{2}{*}{\multicolumn{3}{|c|}{$\begin{array}{l}\text { Descriminação } \\
\text { Ano de publicação do periódico }\end{array}$}} \\
\hline & & \\
\hline 2012 & 01 & $04 \%$ \\
\hline 2013 & 04 & $16 \%$ \\
\hline 2014 & 02 & $08 \%$ \\
\hline 2015 & 03 & $12 \%$ \\
\hline 2016 & 12 & $48 \%$ \\
\hline 2017 & 03 & $12 \%$ \\
\hline \multicolumn{3}{|l|}{ Revistas de publicação } \\
\hline $\begin{array}{l}\text { AMA J Ethics } \\
\text { J Homosex }\end{array}$ & $\begin{array}{l}04 \\
01\end{array}$ & $\begin{array}{l}16 \% \\
04 \%\end{array}$ \\
\hline $\begin{array}{l}\text { J Sex Med } \\
\text { Curr Opin Endocrinol Diabetes Obes }\end{array}$ & $\begin{array}{l}01 \\
04\end{array}$ & $\begin{array}{l}04 \% \\
16 \%\end{array}$ \\
\hline Elsevier Inc J Sex Med & 01 & $04 \%$ \\
\hline Ann Epidemiol & 01 & $04 \%$ \\
\hline Can J Occup Ther & 01 & $04 \%$ \\
\hline Rev enferm UFPE on line & 01 & $04 \%$ \\
\hline Rev méd Chile & 01 & $04 \%$ \\
\hline Arq Bras Endocrinol Metabol & 01 & $04 \%$ \\
\hline Br J Psychiatry & 01 & $04 \%$ \\
\hline Arch Sex Behav & 02 & $08 \%$ \\
\hline PLoS One & 02 & $08 \%$ \\
\hline J Am Board Fam Med & 01 & $04 \%$ \\
\hline Br J Gen Pract & 01 & $04 \%$ \\
\hline BMC Public Health & 02 & $04 \%$ \\
\hline \multicolumn{3}{|l|}{ Profissionais autores } \\
\hline Médicos & 16 & $64 \%$ \\
\hline Sociólogo & 02 & $08 \%$ \\
\hline Psicólogo & 02 & $08 \%$ \\
\hline Terapeuta Ocupacional & 01 & $04 \%$ \\
\hline Advogado & 01 & $04 \%$ \\
\hline Enfermeiro & 01 & $04 \%$ \\
\hline Filósofo & 01 & $04 \%$ \\
\hline Antropólogo & 01 & $04 \%$ \\
\hline \multicolumn{3}{|l|}{ Tipos de Pesquisa } \\
\hline Qualitativa & 11 & $44 \%$ \\
\hline Quantitativa & 09 & $36 \%$ \\
\hline Mista & 02 & $08 \%$ \\
\hline Outro & 03 & $12 \%$ \\
\hline \multicolumn{3}{|l|}{ Tipo de Estudo } \\
\hline Revisão Sistemática & 08 & $32 \%$ \\
\hline Revisão Bibliográfica & 03 & $12 \%$ \\
\hline Revisão Integrativa de Literatura & 04 & $16 \%$ \\
\hline Estudo Clínico & 03 & $12 \%$ \\
\hline Baseado em Evidências. & 02 & $08 \%$ \\
\hline Relato de Experiência. & 02 & $08 \%$ \\
\hline Estudo Etnográfico & 02 & $08 \%$ \\
\hline Estudo Reflexivo & 01 & $04 \%$ \\
\hline \multicolumn{3}{|l|}{ Estado/País } \\
\hline Canadá (EUA) & 06 & $24 \%$ \\
\hline Columbia (EUA) & 02 & $08 \%$ \\
\hline Reino Unido (Inglaterra) & 02 & $08 \%$ \\
\hline Michigan (EUA) & 01 & $04 \%$ \\
\hline Califórnia (EUA) & 01 & $04 \%$ \\
\hline Rockville Pike (EUA) & 01 & $04 \%$ \\
\hline Los Angeles (EUA) & 01 & $04 \%$ \\
\hline Bahia (Brasil) & 01 & $04 \%$ \\
\hline Minneapolis (EUA) & 01 & $04 \%$ \\
\hline Pittisburgh (EUA) & 01 & $04 \%$ \\
\hline Carolina do Norte (EUA) & 01 & $04 \%$ \\
\hline San Juan (México) & 01 & $04 \%$ \\
\hline Atlanta (EUA) & 01 & $04 \%$ \\
\hline Minessota (EUA) & 01 & $04 \%$ \\
\hline Santiago (Chile) & 01 & $04 \%$ \\
\hline São Paulo (Brasil) & 01 & $04 \%$ \\
\hline Okayama (Japão) & 01 & $04 \%$ \\
\hline Albuquerque (NM) & 01 & $04 \%$ \\
\hline
\end{tabular}

Fonte: Banco de dados BVS (LILLACS, SCIELLO, MEDLINE/PUBMED). 
mente. Desta forma, os EUA apresentam maior sensibilidade na produção de pesquisa científica sobre o tema, seguido do Brasil e Reino Unido, que mostram, pela busca realizada, ainda pouco interesse sobre linhas de pesquisa ou sobre o tema. Ressalta-se que no ano de 2018, até o final das buscas nos bancos de dados, não houve publicações de relevância que se aproximassem do tema desse estudo.

Os usuários transexuais estão presentes em todos os níveis do sistema de saúde e geralmente a abordagem cartesiana, utilizada basicamente em todas as esferas de atendimento dos serviços de saúde, os torna invisíveis ou não atende suas necessidades em sua totalidade por desconsiderarem fatores subjetivos que interferem no estabelecimento de relações que primem pela reciprocidade de reconhecimento. Com essa realidade, a partir da reunião das publicações indexadas, se caracterizou a forma como são acolhidos, os focos de atenção e como são reconhecidos e retratados.

O trabalho exploratório acerca de produções indexadas trouxe à tona, a partir das buscas nos bancos de dados, periódicos com temas sobre pessoas transgênero, transexualismo, relacionados à sexualidade, atenção primária em saúde e estratégia de saúde da família. A síntese desses resultados, acrescidos do pensamento por nós valorizado, a saber, o reconhecimento em seus três níveis: do amor, da solidariedade e do direito, inserindo o usuário transexual como cidadão de direitos, autônomos e incorporados dentro do modelo sociocultural. Assim, a partir da síntese dos resultados dos artigos acessados, emergiram núcleos de conteúdo que remeteram às categorias, oportunizando a análise do conteúdo de Bardin, conforme a discussão a seguir.

\section{DISCUSSÃO}

\section{Categoria I: Paradigma Biomédico}

$\mathrm{Na}$ análise dos artigos Art. $\mathrm{I}^{(11)}$, Art. $2^{(12)}$, Art. $3^{(13)}$, Art. $4^{(14)}$,Art. $5^{(15)}$ e Art.6 $6^{(16)}$, percebe-se uma maior preocupação com questões que abordam prioritariamente a área biomédica acerca do tratamento hormonal, dose-resposta e efeitos adversos do tratamento com usuários homens e mulheres transexuais, abordando também a questão de saúde mental e HIV como únicos problemas vivenciados por essa população. Além disso, os autores chegam mesmo a indicar os hábitos de vida como possíveis motivadores das causas de morte desse grupo.Apesar da visão biomédica, cartesiana prevalecer nas produções selecionadas, observa-se indícios de uma preocupação com a qualificação dos médicos, tanto na assistência primária, no primeiro atendimento, quanto em outras esferas, onde descrevem a má formação acadêmica relacionada à questão de gênero e à necessidade de qualificação no processo de transgenitalização, bem como, manutenção do tratamento, onde, em um dos artigos, o usuário chega a dizer que precisou ensiná-lo como o tratar.

Nota-se, na leitura dos artigos mais recentes, que alguns pesquisadores médicos já vislumbram a necessidade de pesquisas que devem ir além de questões biomédicas, dando relevância à inserção social do transexual, assim como, maiores informações sobre os procedimentos e a importância do termo de consentimento informado a fim de garantias legais, tanto para os profissionais quanto para os usuários. Essa nova visão médica é de vital importância, pois alicerça os procedimentos cirúrgicos e hormonais com o contexto sociocultural e subjetividades próprias aos transexuais, fornecendo, assim, um cuidado mais integral.

A abordagem biomédica não pode ser excluída da assistência aos usuários transgênero/transexuais, entretanto, essas não podem ser as únicas apreensões acerca dos cuidados "integrais", há necessidade de se buscar ações em saúde que efetivamente ajudem esses usuários a conceberem mais amplamente sua situação de saúde e condição sociocultural. Esse tipo de cuidado mostra uma visão micro sobre a sexualidade e gênero, o que, por vezes, desqualifica o usuário como um ser autônomo, inserido dentro de um contexto sociocultural de direitos e integralidade, o que, de acordo com os artigos estudados, parece estar caminhando para uma mudança, ainda que lentamente.

O reconhecimento perpassa pela reciprocidade entre usuário e profissional. Ao não ser reconhecido, o usuário não enxerga ou reconhece aquele profissional como capaz de atender as suas necessidades, ocasionando o abandono ao tratamento ou mesmo não procurando o serviço. Isso requer uma mudança de conduta ou ação em saúde, primando pela visão ampliada e saúde integral.

\section{Categoria II: Estigma/invisibilidade}

Os artigos Art7. ${ }^{(17)}$, Art. $8^{(18)}$, Art.9(19), Art.10(20), Art. I I (21),Art. I 2(22),Art. I3 ${ }^{(23)}$ e Art. I4(24)

procuram elucidar o estigma e (in)visibilidade desses usuários dentro das unidades de saúde. Essas questões são extremamente relevantes, pois mostram esses fatores como restritivos ao acesso ou mesmo a procura por um atendimento integral. O estigma sobre o transexual, como um ser indefinido, interfere diretamente no seu tratamento e mesmo na sua invisibilidade/desconsideração dentro dos centros de saúde, causando constrangimento e sofrimento. Porém, ao tratar da questão da invisibilidade, os autores se referem à identificação do ser transgênero tão somente. Esse tema, em que o ser humano, com suas subjetividades, deve ser reconhecido, repercute em autonomia, necessária à tomada de decisão sobre a saúde. De forma contrária, a ausência de autonomia traduz-se por heteronomia, em que a pessoa deixa 
de tomar decisões sobre si, repercutindo em cuidado vertical e prescritivo. Para tanto, há de se considerar as relações intersubjetiva entre profissionais e usuários na esfera do amor, da solidariedade e do direito, contribuindo para a sua autonomia, autorrespeito e autoestima. $O$ reconhecimento se efetiva a partir das relações recíprocas de respeito, corresponsabilidade e valorização dos sujeitos e tratamento justo.

A leitura dos artigos citados mostrou a importância quanto ao quadro emocional dos cidadãos transgêneros/ transexuais em relação à aceitação familiar de sua orientação, assim como do seu círculo de amigos. Os estudos mostraram um alto índice de tentativa de suicídio, muitas vezes com sucesso, principalmente em adolescentes que não se consideram aceitos socialmente, sendo a questão familiar o principal fator de sofrimento, sugerindo inclusive um melhor preparo técnico dos profissionais da saúde para atenuarem essa situação.

Os temas dos quais tratam os artigos podem ser analisados no pensamento honnethiano, pois discutem situações referidas por usuários transexuais que se encontram como indivíduos fora do contexto sociocultural, marginalizados, colocando-os de fora do sistema da equidade e integralidade, se comparados ao que preconiza o SUS. O autor evidencia que os profissionais somente são reconhecidos quando reconhecem os mais vulneráveis (usuários) em sua autonomia, ou seja, como sujeitos de direitos.

\section{Categoria III: Processo de trabalho}

Os artigos categorizados, Art.15(25), Art.16(26), Art. 17(27), Art. 18(28), Art. 19(29), Art.20(30), Art.2I (31),

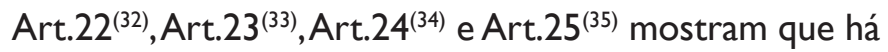
um déficit no que se referem aos processos de trabalho. Em comum nas outras duas categorias, há a afirmação de que a maioria dos profissionais de qualquer esfera da saúde necessita de capacitação para o atendimento desse grupo, mostrando a necessidade emergente em rever os processos de trabalho direcionados aos transexuais masculinos e femininos.

Há consenso, em todos os artigos, de que os profissionais de todas as categorias necessitam rever seus processos de trabalho junto a essa população. Relatam, em suas conclusões, que os próprios profissionais reconhecem suas dificuldades em atuar frente ao transexual e admitem que, mesmo reconhecendo-os como cidadãos, com direito aos cuidados em todas as esferas dos serviços de saúde, não possuem qualificação específica, principalmente no que tange à importância da multidisciplinaridade no acompanhamento desse usuário, assim como no entendimento da construção social do gênero e do corpo, reforçando, assim, uma visão binária na assistência, desconsiderando o subjetivo e a importância das relações intersubjetivas. Considera-se, nessas produções, a importância da inserção do gênero como disciplina obrigatória na grade curricular dos profissionais de saúde.

Para que os cidadãos possam tomar decisões e serem autônomos, as relações entre profissionais e usuários devem incluir a capacitação profissional e sensibilidade para escuta sensível, ativa e diálogo constante. Sem essas condições, os usuários permanecerão ignorando sua situação de saúde, suas possibilidades, ou ainda, as possíveis consequências dos tratamentos implementados. E, sem o diálogo e sensibilidade para as questões subjetivas, os usuários terminam por tomar decisões sobre seu tratamento, respaldados em artigos ou respostas que servem às ideologias que segregam distantes do foco voltado para a sua saúde. Por exemplo, a internet, muitas vezes, oferece informações sem uma base científica, ou sensível às questões socioculturais em que vivem tais usuários. Com isso, emerge a visão utilitarista sobre os sujeitos, afastando-se da condição humana proposta pela gramática moral de reconhecimento Honnethiana.

\section{CONSIDERAÇÕES FINAIS}

Através dos estudos, pode-se observar o cuidado estabelecido sem as considerações subjetivas no que diz respeito a gênero. A construção sociocultural do gênero estabelecida pela sociedade considerando o binarismo homem/mulher heterossexuais como os únicos gêneros aceitáveis e normais, levando o transexual a uma situação de menor valoração social, interfere diretamente em seus direitos como cidadãos tornando-os mais vulneráveis. Essa "menos valia" oriunda do distanciamento dos padrões heteronormativos estabelecidos, muitas vezes, os privam de uma assistência humanizada, de visibilidade e reconhecimento, os transformando em corpos abjetos, ou seja, aqueles não percebidos ou considerados pela sociedade e assim, não necessitando de cuidados de saúde por profissional capacitado. Ressalta-se que essa condição de invisibilidade e não reconhecimento acaba excluindo o transexual não só dos serviços de atendimento à saúde, mas também do ensino educacional e do trabalho formal.

É importante analisar frente os resultados, que a questão levantada a respeito da assistência aos transexuais desprovidas, na maioria das situações, de uma relação empática e holística, não se resume aos programas de saúde dentro do Brasil. Os artigos internacionais dos últimos 2 anos mostram que independente da política de saúde estabelecida naqueles países, o transexual ainda recebe um atendimento muitas vezes baseados em juízo de valores e preconceito, o que mostra o problema em questão indo além das fronteiras nacionais, o que reitera o impacto na assistência a saúde e a relevância das questões levantadas pelos autores. Como vimos, ressalta-se que, embora as questões de atendimento desigual aos 
transexuais foram destacadas no Brasil, a busca realizada mostra que sobre essa população pesa a invisibilidade, heteronomia e preconceito em outros países, não sendo, portanto uma lacuna no cuidado humanizado e inclusivo apenas no Brasil.

Cabe acrescentar que a pesquisa apresentou limitações, não apenas relativas a pouca publicação sobre o tema, mas também referente à própria característica das pesquisas integrativas, que não oferecem possibilidade de generalização, entretanto, por ser tema emergente e pouco explorado, tal revisão oferece, na mesma medida, a possibilidade de reflexão e nova proposta de visibilidade para a atenção em saúde do usuário transexual por aqueles que a acessarem e discutirem a temática. Isso poderá contribuir com a perspectiva da responsabilidade dos profissionais com esses usuários em que pese à contribuição para sua autonomia e reconhecimento.

Nas categorias definidas, a síntese dos resultados das 25 pesquisas publicadas e selecionadas mostra a realidade distante da solidariedade, amor e direito que traduz o reconhecimento dos usuários transexuais. Esses terminam por ser excluído dos seus direitos de acesso ao conhecimento para tomada de decisão, o que interfere na sua autonomia tanto nos resultados de publicações brasileiras quanto estrangeiras.

Embora o tratamento hormonal e o acompanhamento com exames sejam imprescindíveis, a assistência pode e deve ir além, atendendo às reais necessidades de integração desses sujeitos, não mais concebidos com rótulos pré-determinados e sim como sujeitos integrantes da comunidade/sociedade, cuja aceitação e convívio dependem de mu- dança de paradigma, a iniciar-se pela conscientização da real existência do corpo da pessoa transexual, não mais abjeto, mas algo real, palpável e percebido com direitos sociais.

Nesse escopo, compreende-se que a visão contrária ao corpo abjeto pode ser representada por corpos vivos, produtivos, que geram demandas de atendimentos específicos multi e interdisciplinares, oriundos de sua própria transformação, essa tida como única opção de conjunção entre corpo, gênero e sexualidade pelo cidadão transexual para elevação de sua autoestima e sentimento de "estar nesse mundo", obtendo acesso e resolutividade em todas as esferas de atendimento à saúde, estabelecendo assim a integralidade e equidade preconizadas pelas políticas de saúde.

As concepções aqui descritas, baseadas na busca das produções e sustentadas na Teoria Honnethiana, mostram que embora as Políticas de Saúde acolham a camada LGBT é preciso uma nova sensibilização no estabelecimento das relações enfermeiro-transexual, assim como a equipe multidisciplinar.

Ao se analisar a pouca busca pelos serviços de saúde públicos oferecidos, justificados pelo despreparo técnico e atitudes desrespeitosas que levam o constrangimento e afastamento da pessoa transexual, os autores acreditam que esse estudo possa trazer retorno para o campo prático no que se refere ao estabelecimento de uma assistência em que se considere a importância na construção de protocolos assistenciais específicos as demandas dos transexuais, fundamentados em conceitos socioculturais, políticos e antropológicos sobre gênero e suas subjetividades mais sólidas dentro da formação acadêmica. 


\section{REFERÊNCIAS}

I. Brasil. Ministério da Saúde. Portaria $n^{\circ} 399$ de 22 de fevereiro de 2006. Divulga o Pacto pela Saúde 2006 - Consolidação do SUS e aprova as Diretrizes Operacionais do Referido Pacto. Brasília; 2006.

2. Toneli MJ,Amaral M. Sobre travestilidades e políticas públicas: como se produzem os sujeitos da vulnerabilidade. In: Nardi HC, Silveira RS, Machado OS, organizadores. Diversidade Sexual, Relações de Gênero e Políticas Públicas. Porto Alegre: Sulina; 20 I3. p. I5-3I.

3. Brasil. Ministério da Saúde; Secretaria de Gestão Estratégica e Participativa, Departamento de Apoio à Gestão Participativa. Política nacional de saúde integral de lésbicas, gays, bissexuais, travestis e transexuais. Brasília; 2013.

4. Honneth A. Luta por reconhecimento: a gramática moral dos conflitos sociais. Repa L, tradutor. São Paulo: 34;20I I. 296 p.

5. Brasil. Ministério da Saúde; Secretaria Executiva, Núcleo Técnico da Política Nacional de Humanização. Humaniza SUS - Política nacional de humanização: a humanização como eixo norteador das práticas de atenção e gestão em todas as instâncias do SUS. Brasília; 2004.

6. Reis AG, Sauthier M, Soares AM. O cuidado de enfermagem como ampliação das relações de reconhecimento: uma abordagem a partir da perspectiva de Axel Honneth. In: Soares AM, organizador. Outro ângulo: reflexões acerca do humano. Rio de Janeiro: Publit; 2015. p. 93-100.

7. Minayo, Maria Cecília de Souza. $O$ desafio do conhecimento. II ed. São Paulo: Hucitec, 2008.

8. Crossetti MG. Revisão integrativa de pesquisa na enfermagem o rigor cientifico que lhe é exigido [editorial]. Rev gaúch enferm. 2012; 33(2):8-9.

9. Rocha CB. Um pequeno guia ao pensamento, aos conceitos e à obra de Judith Butler. Cad Pagu. 2014; 43:507-I6.

10. Bardin L. Análise de conteúdo. São Paulo: Edições 70; 201 I. $229 \mathrm{p}$.

II. Madeline BD, Radix A, Reisner S. What's in a guideline? Developing collaborative and sound research designs that substantiate best practice recommendations for transgender health care. AMA J Ethics. 2016; 18(II): 1098-106.

12. Murphy TF. Should mental health screening and psychotherapy be required prior to body modification for gender expression? AMA J Ethics. 20 I6; I8(I I): 1079-85.

13. Roblin D, Barzilay J, Tolsma D, Robinson B, Schild L, Cromwell L, et. al.A novel method for estimating transgender status using electronic medical records. Ann Epidemiol [Internet]. 2016 [cited 2017 Dec 10];26(3):198-203. Available from: http://www.annalsofepidemiology.org/article/ SI047-2797(16)30035-7/fulltext. DOI: 10.1016/j.annepidem.2016.01.004.

14. Feldman J, Brown RG, Madeline BD, Hembree W, Meyer W, Meyer-Bahlburg HF, et. al. Priorities for transgender medical and healthcare research. Curr Opin Endocrinol Diabetes Obes [Internet]. 2016 [cited 2017 Dec 10];23(2):180-7.
Available from: https:/www.ncbi.nlm.nih.gov/pmc/articles/ PMC482 I50I/.DOI: I0.1097/MED.000000000000023I.

I5. Costa EM, Mendonça BB. Clinical management of transsexual subjects. Arq Bras Endocrinol Metabol [Internet]. 2014 [cited 2017 Dec I0];58(2): I 88-96.Available from: http://www. scielo.br/pdf/abem/v58n2/0004-2730-abem-58-2-0 I88.pdf.

16. Nakamura A, Watanabe M, Sugimoto M, Sako T, Mahmood $\mathrm{S}$, Kaku $\mathrm{H}$, et. al. Dose-response analysis of testosterone replacement therapy in patients with female to male gender identity disorder. Endocr J [Internet]. 2013 [cited 2017 Dec I0]; 60(3), 275-8I. Available from: https://www.jstage. jst.go.jp/article/endocrj/60/3/60_EJI2-03/9/_article.

17. Cavanaugh T, Hopwood R, Lambert C. Informed consent in the medical care of transgender and gender-nonconforming patients.AMA J Ethics [Interne]. 2016 [cited 2017 Dec I0]; I8(II):I|47-55. Available from: http://journalofethics. ama-assn.org/2016/ I I/sect I- I6I I.html.

18. Lee JG, Ylioia T, Lackey M. Identifying lesbian, gay, bisexual, and transgender search terminology: a systematic review of health systematic reviews. PLoS One [Internet]. 2016 [cited 2017 Dec I0]; I (5):e0I562 I0. Available from: http://journals.plos.org/plosone/article?id=10.137 I/journal. pone.01562I0.

19. Rodríguez-Madera SL, Padilla M, Varas-Diaz N, Neilands T, Vasques Guzzi AC, Florenciani EJ, et. al. Experiences of violence among transgender women in Puerto Rico: an underestimated problem. J Homosex [Internet]. 2017 [cited $2017 \mathrm{Dec} \mathrm{10];64(2):209-17.} \mathrm{Available} \mathrm{from:} \mathrm{https://www.}$ ncbi.nlm.nih.gov/pmc/articles/PMC5546874/. DOI:I0.1080 100918369.2016.1 I74026.

20. Collin L, Reisner SL, Tangpricha V, Goodman M. Prevalence of transgender depends on the "case" definition: a systematic review. J Sex Med [Internet]. 2016 [cited 2017 Dec I0];13(4):6I3-26. Available from: http://www.jsm. jsexmed.org/article/SI 743-6095(I6)00I33-8/fulltext. DOI: I0.1016/j.jsxm.2016.02.00I.

21 . León FM. El reconocimiento legal de la transexualidad en Chile mediante el procedimiento judicial de cambio de nombre:uUn caso de complementariedad epistemológica entre medicina y derecho. Rev méd Chile [Internet]. 2015 [citado 2017 Dic II];|43(8):I0I5-19. Disponible en: http:// www.scielo.cl/pdf/rmc/vl43n8/art08.pdf.

22. Bauer GR, Scheim Al, Pyne J, Travers R, Hammond R. Intervenable factors associated with suicide risk in transgender persons: a respondent driven sampling study in Ontario, Canada. BMC Public Health [Internet]. 2015 [cited 2017 Dec 10];15:525. Available from: https://bmcpublichealth. biomedcentral.com/articles/ | 0. I |86/s | 2889-0 I 5- I867-2. DOI: I0.I |86/s | 2889-0I5-1867-2.

23. Moody C, Smith NG. Suicide protective factors among trans adults. Arch Sex Behav. 2013 Jul;42(5):739-52.

24. Wahlert L, Fiester A. A false sense of security: lesbian, gay, bisexual, and transgender (LGBT) surrogate health care de- 
cision-making rights. J Am Board Fam Med [Internet]. 2013 [cited 2017 Dec 10];26(6):802-4. Available from: http:// www.jabfm.org/content/26/6/802.long. DOI: 10.3122/jabfm.20I3.06.130I30.

25. Giblon R, Bauer GR. Health care availability, quality, and unmet need: a comparison of transgender and cisgender residents of Ontario, Canada. BMC Health Serv Res [Internet]. 2017 [cited 2017 Dec 10];17:283. Available from: https:// www.ncbi.nlm.nih.gov/pmc/articles/PMC5395792/. DOI: |0.1 |86/s | 29|3-017-2226-z.

26. Safer JD, Coleman E, Feldman J, Garofalo R, Hembree W, Ra$\operatorname{dix} A$, et. al. Barriers to healthcare for transgender individuals. Curr Opin Endocrinol Diabetes Obes. 2016;23(2): I68-7I.

27. Bockting W, Coleman E, Deutsch MB, Guillamon A, Meyer I, Meyer W 3rd, Reisner S, Sevelius J, Ettner R. Adult development and quality of life of transgender and gender nonconforming people. Curr Opin Endocrinol Diabetes Obes [Internet]. 2016 [cited 2017 Dec 10];23(2):188-97. Available from: https:/www.ncbi.nlm.nih.gov/pmc/articles/ PMC4809047/. DOI: I0.1097/MED.0000000000000232.

28. Olson-Kennedy J, Cohen-Kettenis PT, Kreukels BP, Meyer -Bahlburg HF, Garofalo R, Meyer W, Rosenthal SM. Research priorities for gender nonconforming/transgender youth: gender identity development and biopsychosocial outcomes. Curr Opin Endocrinol Diabetes Obes [Internet]. 2016 [cited 2017 Dec 10];23(2): I72-179. Available from: https:// www.ncbi.nlm.nih.gov/pmc/articles/PMC4807860/. DOI: 10.1097/MED.0000000000000236.

29. Barrett J. Disorders of gender identity: what to do and who should do it? Br J Psychiatry [Internet]. 2014 [cited 2017 Dec 10];204(2):96-7. Available from: http://bjp.rcpsych.org/ content/204/2/96.long. DOI: I0.I 192/bjp.bp. I I2. 125377.

30. Dietz E, Halem J. How should physicians refer when referral options are limited for transgender patients? AMA J Ethics. 2016;18(I I):1070-8.

3I. Kano M, Silva-Banuelos AR, Sturm R, Willging CE. Stakeholders' recommendations to improve patient-centered "LGBTQ" primary care in rural and multicultural practices. J Am Board Fam Med [Internet]. 2016 [cited 2017 Dec I0];29(I):I56-60. Available from: http://www.jabfm.org/ content/29/1/156.long.

32. Bauer GR, Zong X, Scheim Al, Hammond R, Thind A. Factors impacting transgender patients' discomfort with their family physicians: a respondent-driven sampling survey. PLos One [Internet]. 2015 [cited 20I7 Dec I0]; I0(I2):e0 I 45046.Available from: http://journals.plos.org/plosone/article?id=10.1371/ journal.pone.0145046. DOI: 10.137I/journal.pone.0145046.

33. Zowie D, Siriwardena AN. To be or not to be LGBT in primary health care: health care for lesbian, gay, bisexual, and transgender people. $\mathrm{Br}$ J. Gen. Pract [Internet]. 2012 [cited 2017 Dec 10];62 (602):49I-2. Available from: https:// www.ncbi.nlm.nih.gov/pmc/articles/PMC3426593/. DOI: 10.3399/bjgp I 2X65473 I.

34. Beagan BL, Chiasson A, Fiske CA, Forseth SD, Hosein AC, Myers MR, et. al.Working with transgender clients: learning from physicians and nurses to improve occupational theraPy practice. Can J Occup Ther [Internet]. 2013 [cited 2017 Dec 10];80(2):82-91.Available from:http://journals.sagepub. com/doi/abs/ I0. I I 77/00084 I 74 I 3484450?url_ver=Z39. 88-2003\&rfr_id=ori\%3Arid\%3Acrossref.org\&rfr_dat $=c r$ pub\%3Dpubmed\&. DOI: 0.1 I 77/00084 I74I3484450.

35. Franklin TA, Galvão RA, Boery RN, Sena EL, Yarid SD. Bioethics protection at the lesbians, gays, bisexuals and transgender accessibility. Rev enferm UFPE on line [Internet]. 2016 [cited 2017 Dec 10];10(9):3483-8. Available from: http://pesquisa.bvsalud.org/brasil/resource/pt/bde-30096. 